PROCEEDINGS OF THE

AMERICAN MATHEMATICAL SOCIETY

Volume 130, Number 8, Pages 2335-2342

S 0002-9939(02)06342-6

Article electronically published on January 23, 2002

\title{
A SHARP ESTIMATE ON THE NORM OF THE CONTINUOUS SQUARE FUNCTION
}

\author{
JANINE WITTWER
}

(Communicated by Christopher D. Sogge)

\begin{abstract}
In this paper, we prove that the norm of the continuous square function in $L^{2}(w)$ is bounded linearly in the $A_{2}$ norm of the weight $w$.
\end{abstract}

\section{INTRODUCTION}

The boundedness of singular integral operators in $L^{2}(w)$ for $w \in A_{2}$ has been known for a long time, by the Hunt-Muckenhaupt-Wheeden Theorem. Recently, some of the sharp bounds of these operators in terms of the $A_{2}$ norm of $w$ have been discovered. S. Buckley Buc93 proved that the Hardy-Littlewood maximal operator norm is bounded by $\|w\|_{A_{2}}$ and the square function operator bound is no larger than $\|w\|_{A_{2}}^{3 / 2}$. S. Pott and S. Petermichl [PP] showed that the Hilbert transform norm is bounded by no more than $\|w\|_{A_{2}}^{3 / 2}$. The author proved that the bounds of both the dyadic square function, and the dyadic analog of the Hilbert transform, the martingale transform, are bounded linearly in $\|w\|_{A_{2}}$ Wit00. It is easily seen that each of these operators cannot have a better bound than linear in $\|w\|_{A_{2}}$ (just look at power weights). In this paper, we give a proof that the continuous square function is bounded linearly in $\|w\|_{A_{2}}$.

\section{Notation}

In what follows, $h_{I}$ will denote the normalized Haar function for the dyadic interval $I$, i.e. $h_{I}=\frac{\chi_{I_{l}}-\chi_{I_{r}}}{\sqrt{|I|}}$, where $I_{l}, I_{r}$ denote the left and right children of $I$ respectively. The collection of all dyadic intervals is denoted by $D$. The weight $w$ and its inverse $w^{-1}$ will be $A_{2}$ weights. Let $(f)_{I}$ denote $\frac{1}{\mid T} \int_{I} f(x) d x$ for $I$ a (not necessarily dyadic) interval. Sometimes the parentheses are omitted when it is clear which function we are averaging. $w_{I}^{-1}$ will denote $\frac{1}{\mid T} \int_{I} \frac{1}{w(x)} d x$. Let $\mu(I)=w_{I} w_{I}^{-1}$ and $\|w\|_{A_{2}}=\sup _{I} \mu(I) .\langle,\rangle_{w}$ will denote the inner product in $L^{2}(w(x) d x)$. If the subscript is omitted, $w=1$. Let $\phi$ be a function with $\int \phi=0 . \phi^{I}=\frac{1}{\sqrt{B}} \phi\left(\frac{x-A}{B}\right)$, where $A$ is the starting point of the interval $I$, and $B$ is the length $|I|$. Similarly, $\phi_{t}(x)=\frac{1}{\sqrt{t}} \phi\left(\frac{x}{t}\right)$. In this paper, $c$ will denote a constant, not necessarily the same at each occurrence, which is independent of $f$ and $w$, but may depend on $\phi$.

Received by the editors September 11, 2000 and, in revised form, March 9, 2001.

2000 Mathematics Subject Classification. Primary 42A50.

(C)2002 American Mathematical Society 
The continuous square function will be denoted by $S f$ and is defined by

$$
S f(x)=\left(\int_{\Gamma(x)}\left|f \star \phi_{t}(y)\right|^{2} \frac{d y d t}{t^{3}}\right)^{1 / 2},
$$

where $\Gamma(x)=\{(y, t):|y-x|<t\}$.

The dyadic square function takes the form

$$
S_{d} f(x)=\left(\sum_{I \in D, x \in I}\left\langle f, h_{I}\right\rangle^{2} \frac{1}{|I|}\right)^{1 / 2}
$$

and the wavelet square function is

$$
S_{\phi, d} f(x)=\left(\sum_{I \in D, x \in I}\left\langle f, \phi^{I}\right\rangle^{2} \frac{1}{|I|}\right)^{1 / 2} .
$$

\section{Statements of theOREmS}

Theorem 3.1. Let $\phi(x)$ be compactly supported in $[0,1], C^{1}$, such that $\left\{\phi^{I}\right\}_{I}$ is an orthonormal basis of $L^{2}(d x)$, and $\int \phi=0$. Then

$$
\left\|S_{\phi, d} f\right\|_{L^{2}(w)} \leq c\|w\|_{A_{2}}\|f\|_{L^{2}(w)}
$$

for $w \in A_{2}$ and $f \in L^{2}(w)$.

Theorem 3.2. Let $\phi(x)$ be compactly supported in $[0,1], C^{1}$, such that $\left\{\phi^{I}\right\}_{I}$ is an orthonormal basis of $L^{2}(d x)$, and $\int \phi=0$. Then

$$
\|S f\|_{L^{2}(w)} \leq c\|w\|_{A_{2}}\|f\|_{L^{2}(w)}
$$

for $w \in A_{2}$ and $f \in L^{2}(w)$.

Note. Such $\phi$ are called wavelets. See Dau92 for a well written discussion of wavelets and information on how to construct $C^{r}$ wavelets with compact support.

\section{ProOFs}

Proof (Theorem[3.1). By [FP97] we know that

$$
\left\|S_{d} f\right\|_{L^{2}(w)}=\left\|\left(\sum_{I \in D, x \in I} \frac{1}{|I|}\left\langle f, h_{I}\right\rangle^{2}\right)^{1 / 2}\right\|_{L^{2}(w)} \geq c\|w\|_{A_{2}}^{-1 / 2}\|f\|_{L^{2}(w)} .
$$

To prove the theorem, we shall prove that $S_{d}(x) \leq C S_{\phi, d}(x)$, and that therefore the norm of $S_{\phi, d}$ is bounded below by $c\|w\|_{A_{2}}^{1 / 2}$. Once we know the lower bound, we can derive the upper bound using the same proof used in [Wit00]. 
Step 1 (see also Wil89]): Show $S_{d} f(x) \leq c S_{\phi, d} f(x)$.

$f(x)=\sum_{J \in D}\left\langle f, \phi^{J}\right\rangle \phi^{J}$. Inserting this expression for $f$ into the definition of $S_{d} f(x)$, we have

$$
\begin{aligned}
S_{d}^{2} f(x) & =\sum_{I \in D, x \in I}\left(\left\langle\sum_{J}\left\langle f, \phi^{J}\right\rangle \phi^{J}, h_{I}\right\rangle\right)^{2} \frac{1}{|I|} \\
& =\sum_{I \in D, x \in I}\left(\sum_{J}\left\langle f, \phi^{J}\right\rangle\left\langle\phi^{J}, h_{I}\right\rangle\right)^{2} \frac{1}{|I|} .
\end{aligned}
$$

Let $\lambda_{J}=\left\langle f, \phi^{J}\right\rangle$. We need to estimate

$$
\sum_{I \in D} \chi_{I}(x)\left(\sum_{J: I \subset J} \lambda_{J}\left\langle\phi^{J}, h_{I}\right\rangle\right)^{2} \frac{1}{|I|},
$$

where we only sum over $J: I \subset J$, since otherwise the inner product is 0 , as $\int \phi=0$. Apply Cauchy-Schwarz to get

$$
S_{d}^{2} f(x) \leq \sum_{I} \frac{\chi_{I}(x)}{|I|}\left(\sum_{J: I \subset J} \lambda_{J}^{2} \frac{|I|^{3}}{|J|^{2}}\right)\left(\sum_{J: I \subset J}\left\langle\phi^{J}, h_{I}\right\rangle^{2} \frac{|J|^{2}}{|I|^{3}}\right) .
$$

Let us estimate $\left\langle\phi^{J}, h_{I}\right\rangle$.

Let $M$ satisfy $\|\phi\|_{\infty}+\left\|\phi^{\prime}\right\|_{\infty} \leq M$. Then

$$
\left\langle\phi^{J}, h_{I}\right\rangle=\frac{1}{|I|}\left(\int_{I_{l}} \phi^{J}-\int_{I_{r}} \phi^{J}\right) \sqrt{|I|}=\left(\phi^{J}\left(\xi_{I_{l}}\right)-\phi^{J}\left(\xi_{I_{r}}\right)\right) \sqrt{|I|} / 2 \leq c M \frac{|I|^{3 / 2}}{|J|^{3 / 2}},
$$

since $\left|\phi^{J^{\prime}}\right| \leq \frac{M}{|J|^{3 / 2}}$. Therefore, $S_{d}^{2} f(x)$ is bounded by

$$
\begin{aligned}
& c M^{2} \sum_{I} \frac{\chi_{I}(x)}{|I|}\left(\sum_{J: I \subset J} \lambda_{J}^{2} \frac{|I|^{3}}{|J|^{2}}\right)\left(\sum_{J: I \subset J} \frac{1}{|J|}\right) \\
& \quad \leq c M^{2} \sum_{I} \frac{\left(\chi_{I}(x)\right)^{2}}{|I|}\left(\sum_{J: I \subset J} \lambda_{J}^{2} \frac{|I|^{2}}{|J|^{2}}\right) \\
& \quad \leq c M^{2} \sum_{J} \frac{\chi_{J}(x)}{|J|^{2}} \lambda_{J}^{2} \sum_{I: I \subset J}|I| \chi_{I} \leq c M^{2} \sum_{J: x \in J} \frac{\lambda_{J}^{2}}{|J|}=c M^{2} S_{\phi, d}^{2} f(x),
\end{aligned}
$$

where we have used the fact that $\chi_{I} \leq \chi_{J}$. Therefore, $S_{d} f(x) \leq c M S_{\phi, d} f(x)$.

Step 2: Derive the upper bound from the lower bound.

Let

$$
f_{I}=\frac{\phi^{I}}{\left(w_{I}\right)^{1 / 2}}
$$

and

$$
g_{I}=\phi^{I}\left(w_{I}\right)^{1 / 2} w^{-1} .
$$

Then $\left\langle f_{I}, g_{J}\right\rangle_{w}=1$ if $I=J$ and 0 otherwise. Let $f \in L^{2}(w)$.

$$
\left\|S_{\phi, d}\right\|_{L^{2}(w)}=\left(\sum_{I \in D}\left\langle f, \phi^{I}\right\rangle^{2} w_{I}\right)^{1 / 2}=\left(\sum_{I \in D}\left\langle f, g_{I}\right\rangle_{w}^{2}\right)^{1 / 2} .
$$


In that notation, the estimate to prove becomes

$$
\sum_{I \in D}\left\langle f, g_{I}\right\rangle_{w}^{2} \leq c\|w\|_{A_{2}}^{2}\|f\|_{L^{2}(w)}^{2} .
$$

We will bound this by first showing that

$$
\sum_{I \in D}\left\langle f, f_{I}\right\rangle_{w}^{2} \leq c\|w\|_{A_{2}}\|f\|_{L^{2}(w)}^{2},
$$

from which the desired inequality can be extracted by algebraic manipulations.

By Step 1, we know the following:

$$
\|f\|_{L^{2}(w)} \leq c\|w\|_{A_{2}}{ }^{1 / 2}\left(\sum_{I \in D}\left\langle f, g_{I}\right\rangle_{w}^{2}\right)^{1 / 2} .
$$

This allows us to compute the norm of the sequence valued operator $(J g)_{I}=$ $\left\langle g, f_{I}\right\rangle_{w}$ from $L^{2}(w)$ to $l^{2}$ :

$$
\begin{aligned}
& \sup _{\|g\|_{L^{2}(w)}=1} \sup _{\left\|\left\{k_{I}\right\}\right\|_{L^{2}}=1} \sum_{I \in D} k_{I} \int g f_{I} w \\
& =\sup _{\|g\|_{L^{2}(w)}=1\left\|\left\{k_{I}\right\}\right\|_{l^{2}}=1} \int g \sum_{I \in D} k_{I} f_{I} w
\end{aligned}
$$

by Cauchy-Schwarz, and the above inequality,

$$
\begin{aligned}
& \leq \sup _{\left\|\left\{k_{I}\right\}\right\|_{l^{2}}=1} \sup _{\|g\|_{L^{2}(w)}=1}\left\|\sum_{I \in D} k_{I} f_{I}(x)\right\|_{L^{2}(w)}\|g\|_{L^{2}(w)} \\
& \leq c\|w\|_{A_{2}}{ }^{1 / 2} \sup _{\left\|\left\{k_{I}\right\}\right\|_{l^{2}}=1}\left[\sum_{J \in D}\left\langle\left(\sum_{I \in D} k_{I} f_{I}\right), g_{J}\right\rangle_{w}^{2}\right]^{1 / 2} \\
& =c\|w\|_{A_{2}}{ }^{1 / 2} \sup _{\left\|\left\{k_{I}\right\}\right\|_{l^{2}}=1}\left(\sum_{I \in D}{k_{I}}^{2}\right)^{1 / 2}=c\|w\|_{A_{2}}{ }^{1 / 2} .
\end{aligned}
$$

This means that

$$
\sum_{I \in D}\left\langle g, f_{I}\right\rangle_{w}^{2} \leq c\|w\|_{A_{2}}\|g\|_{L^{2}(w)}^{2}
$$

for every weight in $A_{2}$. In particular, this is true for the weight $w^{-1}$ :

$$
\sum_{I \in D}\left\langle g, \frac{\phi^{I}}{\left(w_{I}^{-1}\right)^{1 / 2}}\right\rangle_{w^{-1}}^{2} \leq c\|w\|_{A_{2}}\|g\|_{L^{2}\left(w^{-1}\right)}^{2} .
$$

Let $g^{*}=g w^{-1}$. Then this becomes

$$
\sum_{I \in D}\left\langle g^{*}, \frac{\phi^{I}}{\left(w_{I}^{-1}\right)^{1 / 2}}\right\rangle_{d x}^{2} \leq c\|w\|_{A_{2}}\left\|g^{*}\right\|_{L^{2}(w)}^{2} .
$$

The left-hand side can be rewritten as

$$
\sum_{I \in D}\left\langle g^{*}, \phi^{I}\left(w_{I}\right)^{1 / 2} w w^{-1}\right\rangle_{d x}^{2} \frac{1}{\mu(I)} .
$$


So finally, we have the inequality

$$
\sum_{I \in D}\left\langle g^{*}, g_{I}\right\rangle_{w}^{2} \leq c\|w\|_{A_{2}}^{2}\left\|g^{*}\right\|_{L^{2}(w)}^{2},
$$

which is what we needed to prove.

Proof (Theorem 3.2). We will prove Theorem 3.2 using Theorem 3.1.

We will proceed as follows: First we will prove that the upper bound on the wavelet square function is still valid if we translate and dilate the dyadic grid. Then we write the continuous square function as an average of the translated/dilated operators over a continuum of translations and dilations.

Recall that $\phi^{I}=\frac{1}{\sqrt{B}} \phi\left(\frac{x-A}{B}\right)$, where $A$ is the starting point of the interval $I$, and $B$ is the length $|I|$. By Theorem 3.1 .

$$
\left\|\left(\sum_{I \in D, x \in I} \frac{1}{|I|}\left\langle f, \phi^{I}\right\rangle^{2}\right)^{1 / 2}\right\|_{L^{2}(w)} \leq c\|w\|_{A_{2}}\|f\|_{L^{2}(w)} .
$$

For $\alpha \in \mathbb{R}$ and $1 \leq \beta<2$, define

$$
D_{\alpha, \beta}=\left\{I: I=\left[\beta k 2^{i}+\alpha, \beta(k+1) 2^{i}+\alpha\right) ; i, k \in \mathbb{Z}\right\} .
$$

Lemma 4.1. For fixed $\alpha$ and $\beta$, let

$$
S_{\phi, \alpha, \beta} f(x)=\left(\sum_{I \in D_{\alpha, \beta}, x \in I} \frac{1}{|I|}\left\langle f, \phi^{I}\right\rangle^{2}\right)^{1 / 2} .
$$

Then,

$$
\left\|S_{\phi, \alpha, \beta} f(x)\right\|_{L^{2}(w)} \leq c\|w\|_{A_{2}}\|f\|_{L^{2}(w)},
$$

where $c$ is independent of $\alpha$ and $\beta$.

Proof.

$$
\begin{aligned}
& \left\|\left(\sum_{I \in D_{\alpha, \beta}, x \in I} \frac{1}{|I|}\left\langle f, \phi^{I}\right\rangle^{2}\right)^{1 / 2}\right\|_{L^{2}(w)}^{2}=\sum_{I \in D_{\alpha, \beta}}\left\langle f, \phi^{I}\right\rangle^{2} w_{I} \\
& =\sum_{i, k}\left(\int f(u) \frac{\phi\left(\frac{u-\left(\beta k 2^{i}+\alpha\right)}{\beta 2^{i}}\right)}{\sqrt{\beta 2^{i}}} d u\right)^{2} \frac{1}{\beta 2^{i}} \int_{\left[\beta k 2^{i}+\alpha, \beta(k+1) 2^{i}+\alpha\right]} w(u) d u .
\end{aligned}
$$

Change variables by letting $\tau=(u-\alpha) / \beta$.

The above becomes

$$
\begin{aligned}
\sum_{i, k} & \left(\int f(\beta \tau+\alpha) \phi\left(\frac{\tau-k 2^{i}}{2^{i}}\right) \frac{\beta d \tau}{\sqrt{\beta 2^{i}}}\right)^{2} \frac{1}{2^{i}} \int_{\left[k 2^{i},(k+1) 2^{i}\right]} w(\beta \tau+\alpha) d \tau \\
& =\sum_{I \in D}\left\langle f(\beta \tau+\alpha), \phi^{I}(\tau)\right\rangle_{d \tau}^{2} \beta \frac{1}{|I|} \int_{I} w(\beta \tau+\alpha) d \tau
\end{aligned}
$$


which, by the boundedness of the operator with regular dyadic cubes, is

$$
\leq \beta c\|w\|_{A_{2}}^{2} \int f^{2}(\beta \tau+\alpha) w(\beta \tau+\alpha) d \tau=c\|w\|_{A_{2}}^{2}\|f\|_{L^{2}(w)}^{2}
$$

using the reverse change of variables.

Note. $c\|w(\beta \tau+\alpha)\|_{A_{2}}=c\|w\|_{A_{2}}$, since dilation and translation do not affect the $A_{2}$ norm.

Let $N$ be a measurable subset of the $\alpha-\beta$ plane. Then $\left\|S_{\phi, \alpha, \beta} f(x)\right\|_{L^{2}\left(N, \frac{d \alpha d \beta}{N}\right)}$ is a function of $x$, with norm in $L^{2}(w)$, and furthermore,

$$
\|\| S_{\phi, \alpha, \beta} f(x)\left\|_{L^{2}\left(N, \frac{d \alpha d \beta}{|N|}\right)}\right\|_{L^{2}(w)}=\|\| S_{\phi, \alpha, \beta} f(x)\left\|_{L^{2}(w)}\right\|_{L^{2}\left(N, \frac{d \alpha d \beta}{|N|}\right)} \leq c\|w\|_{A_{2}}\|f\|_{L^{2}(w)}
$$

for any $N$.

Let $N$ be $[-M, M] \times[1,2]$ for a fixed real $M$. For this $N$,

$$
\begin{aligned}
& \left\|S_{\phi, \alpha, \beta} f(x)\right\|_{L^{2}\left(N, \frac{d \alpha d \beta}{\mid N}\right)}^{2} \\
& =\frac{1}{2 M} \int_{-M}^{M} \int_{1}^{2} \sum_{I \in D_{\alpha, \beta}, x \in I}\left(\int f(u) \frac{\phi\left(\frac{u-\left(\beta k 2^{i}+\alpha\right)}{\beta 2^{i}}\right)}{\sqrt{\beta 2^{i}}} d u\right)^{2} \frac{1}{\beta 2^{i}} d \beta d \alpha \\
& =\sum_{i} \int_{1}^{2} \frac{1}{2 M} \int_{-M}^{M}\left(\int f(u) \frac{\phi\left(\frac{u-\left(\beta k 2^{i}+\alpha\right)}{\beta 2^{i}}\right)}{\sqrt{\beta 2^{i}}} d u\right)^{2} \frac{1}{\beta 2^{i}} d \alpha d \beta
\end{aligned}
$$

where $k=k(\alpha, \beta, i, x)$, namely the unique $k$ such that $x \in\left[k 2^{i} \beta+\alpha,(k+1) 2^{i} \beta+\alpha\right)$.

Now break up the integral $\int_{-M}^{M}$ into chunks of $\alpha$ for which $k$ is the same, i.e. $\alpha \in\left(x-2^{i} \beta(k+1), x-2^{i} \beta k\right]$. Obviously, we cannot always break up the integral exactly into such chunks (and for some $i$, there will be no chunks at all), so we only take the whole chunks, and throw away the end pieces, since they are positive anyway. Therefore the above is bigger than

$$
\sum_{i} \int_{1}^{2} \frac{1}{2 M} \sum_{k=A(M, \beta, i, x)}^{B(M, \beta, i, x)} \int_{x-2^{i} \beta(k+1)}^{x-2^{i} \beta k}\left(\int f(u) \frac{\phi\left(\frac{u-\left(\beta k 2^{i}+\alpha\right)}{\beta 2^{i}}\right)}{\sqrt{\beta 2^{i}}} d u\right)^{2} \frac{1}{\beta 2^{i}} d \alpha d \beta
$$

where a calculation shows that $A(M, \beta, i, x)=$ the closest integer bigger than $\frac{x-M}{2^{i} \beta}$ and $B(M, \beta, i, x)=$ the closest integer small than $\frac{x+M}{2^{i} \beta}-1$. The sum is understood to be 0 if $B<A$.

Now change variables $y=\alpha+k 2^{i} \beta$ and $t=2^{i} \beta$. This change of variables has determinant $2^{-i}$, which is proportional to $\frac{1}{t}$. (Remember that $\beta \in[1,2]$.)

The resulting integral is

$$
\begin{gathered}
\sum_{i} \int_{2^{i}}^{2^{i+1}} \frac{1}{2 M} \sum_{k=A(M, t, x)}^{B(M, t, x)} \int_{x-t}^{x}\left(\int f(u) \frac{\phi\left(\frac{u-y}{t}\right)}{\sqrt{t}} d u\right)^{2} \frac{1}{t^{2}} d y d t \\
=\int_{\mathbb{R}^{+}} \frac{1}{2 M} \sum_{k=A(M, t, x)}^{B(M, t, x)} \int_{x-t}^{x}\left|f \star \tilde{\phi}_{t}(y)\right|^{2} \frac{1}{t^{2}} d y d t,
\end{gathered}
$$

where $\tilde{\phi}(x)$ denotes $\phi(-x)$. 
Notice that $k$ no longer appears in the integrand, so we can replace $\sum_{k=A(M, t, x)}^{B(M, t, x)}$ by $\max \{0, B(M, t, x)-A(M, t, x)+1\} . B(M, t, x)-A(M, t, x)+1 \geq \frac{M+x}{t}-\frac{x-M}{t}-$ $2=\frac{2 M}{t}-2$, so if $M \geq t$ (and certainly if $M / 2 \geq t$ ), the second term of the max is larger. Therefore the above is at least

$$
\begin{gathered}
\int_{0<t \leq \frac{M}{2}} \frac{1}{2 M}\left(\frac{2 M}{t}-2\right) \int_{x-t}^{x}\left|f \star \tilde{\phi}_{t}(y)\right|^{2} \frac{1}{t^{2}} d y d t \\
\quad \geq c \int_{0<t \leq \frac{M}{2}}\left(\frac{2}{t}-\frac{1}{t}\right) \int_{x-t}^{x}\left|f \star \tilde{\phi}_{t}(y)\right|^{2} \frac{1}{t^{2}} d y d t \\
\quad=c \int_{0<t \leq \frac{M}{2}} \int_{x-t}^{x}\left|f \star \tilde{\phi}_{t}(y)\right|^{2} \frac{1}{t^{3}} d y d t .
\end{gathered}
$$

To summarize, we have proven that

$$
\begin{aligned}
& \left\|S_{\phi, \alpha, \beta} f(x)\right\|_{L^{2}\left([-M, M] \times[1,2], \frac{d \alpha d \beta}{2 M}\right)}^{2} \\
& \quad \geq c \int_{0<t \leq \frac{M}{2}} \int_{x-t}^{x}\left|f \star \tilde{\phi}_{t}(y)\right|^{2} \frac{1}{t^{3}} d y d t,
\end{aligned}
$$

uniformly in $M$. Therefore,

$$
\begin{aligned}
c\|w\|_{A_{2}}\|f\|_{L^{2}(w)} & \geq\|\| S_{\phi, \alpha, \beta} f(x)\left\|_{L^{2}\left([-M, M] \times[1,2], \frac{d \alpha d \beta}{2 M}\right)}\right\|_{L^{2}(w)} \\
& \geq\left\|\left(c \int_{0<t \leq \frac{M}{2}} \int_{x-t}^{x}\left|f \star \tilde{\phi}_{t}(y)\right|^{2} \frac{1}{t^{3}} d y d t\right)^{1 / 2}\right\|_{L^{2}(w)}
\end{aligned}
$$

uniformly in $M$. Take the limit as $M \rightarrow \infty$ to get

$$
\left\|\left(\int_{\mathbb{R}^{+}} \int_{x-t}^{x}\left|f \star \tilde{\phi}_{t}(y)\right|^{2} \frac{1}{t^{3}} d y d t\right)^{\frac{1}{2}}\right\|_{L^{2}(w)} \leq c\|w\|_{A_{2}}\|f\|_{L^{2}(w)} .
$$

By considering $f(-u)$ instead of $f(u)$, and following the minus sign through, it is also easily seen that

$$
\left\|\left(\int_{\mathbb{R}^{+}} \int_{x}^{x+t}\left|f \star \tilde{\phi}_{t}(y)\right|^{2} \frac{1}{t^{3}} d y d t\right)^{\frac{1}{2}}\right\|_{L^{2}(w)} \leq c\|w\|_{A_{2}}\|f\|_{L^{2}(w)},
$$

which proves that

$$
\|S f(x)\|_{L^{2}(w)} \leq c\|w\|_{A_{2}}\|f\|_{L^{2}(w)} .
$$

\section{REFERENCES}

[Buc93] Stephen M. Buckley, Estimates for operator norms on weighted spaces and reverse Jensen inequalities, Trans. Amer. Math. Soc. 340 (1993), no. 1, 253-272. MR 94a:42011

[Dau92] I. Daubechies, Ten lectures on wavelets, CBMS-NSF Regional Conference Series in Applied Mathematics, 61, Philadelphia, 1992. MR 93e:42045

[FP97] R. Fefferman and J. Pipher, Multiparameter operators and sharp weighted inequalities, Amer. J. Math. 119 (1997), no. 2, 337-369. MR 98b:42027

$[\mathrm{PP}] \quad \mathrm{S}$. Petermichl and S. Pott, An estimate for weighted hilbert transform via square functions, personal communication. 
[Wil89] J. Michael Wilson, Weighted norm inequalities for the continuous square function, Trans. Amer. Math. Soc. 314 (1989), no. 2, 661-692; Erratum, Trans. Amer. Math. Soc. 321 (1990), 415. MR 91e:42025 MR 91e:42026

[Wit00] J. Wittwer, A sharp estimate on the norm of the martingale transform, Math. Res. Lett. 7 (2000), no. 1, 1-12. MR 2001e:42022

Department of Mathematics, Williams College, Williamstown, Massachusetts 01267

E-mail address: jwittwer@williams.edu 